\title{
1 Genetic variance in fitness and its cross-sex covariance 2 predict adaptation during experimental evolution.
}

\author{
Short running title: Adaptive potential to new environments
}

Authors: Eva L. Koch ${ }^{1,2}$, Sonja H. Sbilordo ${ }^{1}$, and Frédéric Guillaume ${ }^{1}$

Affiliations: ${ }^{1}$ Department of Evolutionary Biology and Environmental Studies, University of Zürich, Winterthurerstr. 190, 8057 Zürich, Switzerland

${ }^{2}$ Department of Animal and Plant Science, University of Sheffield, Western Bank, Sheffield S10 2TN,

United Kingdom

\section{Corresponding author:}

Frédéric Guillaume

phone: +41446356623

guillaume.fred@gmail.com

\section{Abstract}

In presence of rapid environmental changes, it is of particular importance to assess the adaptive potential of populations, which is mostly determined by the additive genetic variation $\left(V_{A}\right)$ in fitness. In this study we used Tribolium castaneum (red flour beetles) to investigate its adaptive potential in three new environmental conditions (Dry, Hot, Hot-Dry). We tested for potential constraints that might limit adaptation, including negative genetic covariance between female and male fitness. Based on $V_{A}$ estimates for fitness, we expected the highest relative fitness increase in the most stressful condition Hot-Dry and similar increases in single stress conditions Dry and Hot. High adaptive potential in females in Hot was reduced by a negative covariance with male fitness. We tested adaptation to the three conditions after 20 generations of experimental evolution and found that observed adaptation mainly matched our predictions. Given that body size is commonly used as a proxy for fitness, we also tested how this trait and its genetic variance (including non-additive genetic variance) were impacted by environmental stress. In both traits, variances were sex and condition dependent, but they differed in their variance composition, cross-sex and cross-environment genetic covariances, as well as in the environmental impact on $\mathrm{V}_{\mathrm{A}}$. 
25 Keywords: fundamental theorem of natural selection, non-additive genetic effects, heritability, quantitative

26 genetics, evolvability

\section{Introduction}

29 Environmental changes pose a substantial risk of extinction to many organisms (Thomas et al. 2004; Parmesan

30 2006). Predicting whether a population is able to persist is therefore of crucial importance. Species may adapt

31 via plastic or genetic changes. Plastic changes, e.g. physiological or behavioural adjustments, allow individuals

32 to cope with stressful conditions (Charmantier et al. 2008). However, plastic responses are often costly and

33 thus likely limited (Houle 1992; DeWitt et al. 1998; de Jong 2005; Valladares et al. 2007; Pfennig et al. 2010;

34 Snell-Rood et al. 2010; Sokolova et al. 2012). It is also not clear whether they are sufficient to compensate

35 negative effects of environmental changes (Duputié et al. 2015; Arnold et al. 2019). Instead, fast 36 environmental shifts may require rapid adaptation by genetic evolution on short time scales. In this case, the 37 standing genetic variation already present in the population is of particular importance (Kellermann et al. 38 2006; Bell and Gonzalez 2009). Adaptive evolution proceeds through different individual contributions to the 39 next generation, i.e. differences in their fitness. Importantly, this variation in individual fitness has to be at 40 least partly due to some underlying genetic variation in order to change the genetic composition of a 41 population. Usually, only variance due to additive genetic effects $\left(V_{A}\right)$ is considered since additive effects are 42 inherited from parents to offspring and determine the response to selection. $\mathrm{V}_{\mathrm{A}}$ of relative fitness gives the 43 expected increase of fitness in the next generation (Fisher 1930; Price 1972; Falconer and MacKay 1996). Thus, 44 the existence of $\mathrm{V}_{\mathrm{A}}$ in fitness is a prerequisite for adaptation and can be used as an overall estimate of a 45 population's capacity to adapt, or its evolvability (Houle 1992; Hansen et al. 2011; Shaw and Shaw 2014).

47 A common and intuitive expectation is that $V_{A}$ in fitness should be low because selection depletes genetic 48 variation in adapted populations. Accordingly, it was found that heritability $\left(\mathrm{h}^{2}\right.$, proportion of $\mathrm{V}_{\mathrm{A}}$ in total 
49 variance $V_{p}$ ) was lower in traits more closely associated with fitness (Mousseau and Roff 1987; Kruuk et al.

50 2000; Merilä and Sheldon 2000; Teplitsky et al. 2009; Wheelwright et al. 2014). However, $\mathrm{h}^{2}$ is not ideal for

51 estimating $V_{A}$ in fitness and the evolutionary potential of a population (Hansen et al. 2011; Wheelwright et al.

52 2014; Morrissey and Bonnet 2019) since low $\mathrm{h}^{2}$ is often due to higher environmental variance (Schluter et al.

53 1991; Merilä and Sheldon 1999). Many populations may also not be at an evolutionary equilibrium (Shaw and

54 Shaw 2014) in environments that have been recently changed by human impacts (Fugère and Hendry 2018).

55 Estimates of $\mathrm{V}_{\mathrm{A}}$ in fitness are scarce, especially in natural populations and tend to show large heterogeneity in

56 the estimates of $V_{A}$ for lifetime fitness (e.g., in vertebrate populations: Kruuk et al., 2000; Merilä \& Sheldon,

57 2000; McCleery et al., 2004; McFarlane et al., 2014). Although it is predicted that some species might not have

58 the evolutionary potential to adapt to shifting environmental conditions (Etterson and Shaw 2001), some

59 studies found substantial amounts of $\mathrm{V}_{\mathrm{A}}$ in fitness in wild populations. Similarly, laboratory studies have

60 reported significant $V_{A}$ for fitness related traits in D. melanogaster (Gardner et al. 2005; Fry 2008; Long et al.

$612009)$.

62

63 Despite existing $V_{A}$ in fitness traits, evolution to new conditions can be constrained by antagonistic pleiotropy if

64 alleles influence several fitness components but with opposite effects. This leads to trade-offs since one

65 component cannot be optimized without reducing the other, e.g. fecundity and life length (Roff 2000). Another

66 example of trade-offs is sexual antagonism (Foerster et al. 2007; Bonduriansky and Chenoweth 2009; Delcourt

67 et al. 2009; Kirkpatrick 2009; Poissant et al. 2010; Calsbeek et al. 2015; Connallon and Hall 2016). Fitness

68 optima might often differ between males and females. However, sharing a great part of their genome

69 constrains independent evolution and limits adaptation when selection in the two sexes is opposite to the

70 genetic correlation. Such a constraint should be revealed by negative genetic correlations between male and

71 female fitness. Similar constraints may appear when adaptation to certain environmental conditions (e.g.,

72 elevated temperature) trades-off with adaptation to other conditions. Environmental changes often include 
73 simultaneous changes in several variables. For short-term stress exposure, effects like cross-tolerance and

74 hardening (i.e., resistance to one stress develops after exposure to another stress) were observed, and it is

75 known that some stress responses rely on the same physiological mechanisms (Bubliy et al. 2012).

76 Evolutionary adaptation to different stressors involving the same pathways may thus lead to correlated

77 resistance to another stressor (Bubliy and Loeschcke 2005; Sikkink et al. 2015). However, examples of local

78 adaptation suggest that selection may often favour different genotypes in different conditions (Hereford

79 2009). Immediately after exposure to an environmental change, genetic correlations between fitness in

80 different conditions can inform us to what extent the genetic basis of fitness is shared between environments.

81 If we observe negative genetic covariances between fitness in different conditions, it is likely that alleles

82 providing fitness benefits in one condition become detrimental in another. Adaptation when both stress

83 factors are experienced at the same time can then be limited.

84

85 Changes in environmental conditions are also well known to affect genetic variances (Sgrò and Hoffmann 1998;

86 Hoffmann and Merilä 1999; Rowiński and Rogell 2017), and covariances (Simons and Roff 1996; Sgrò and

87 Hoffmann 2004; Wood and Brodie 2015), including cross-sex genetic covariance (Delcourt et al. 2009; Poissant

88 et al. 2010; Punzalan et al. 2014). This can have substantial implications for evolutionary potential (Wilson et

89 al. 2006; Husby et al. 2011), since the environmental shift that imposes a risk of extinction can at the same

90 time either increase $V_{A}$ for fitness (Shaw and Shaw, 2014) or reduce the evolutionary potential to adapt to this

91 new condition (Wood and Brodie 2016). To fully understand the impact of environmental change on a

92 population's persistence it is therefore essential to know how genetic variances change in different

93 environments and to identify potential constraints.

95 The fundamental importance of $\mathrm{V}_{\mathrm{A}}$ of fitness for predicting contemporary evolution (Shaw and Etterson 2012;

96 Hendry et al. 2018) and recent statistical advances in quantitative genetics have fostered great interest in 97 estimating the adaptive capacity of wild populations (Charmantier et al. 2014). Even more so with the recent 
98 progress in using genomic markers to infer genetic resemblance among individuals (Gienapp et al. 2017;

99 Perrier et al. 2018). These advances open up new perspectives for applications of classical quantitative genetic

100 and genomic tools in wild populations, addressing important questions regarding populations' persistence

101 under environmental change (Waldvogel et al. 2020). However, so far relatively little information exists about

102 the predictive value of $\mathrm{V}_{\mathrm{A}}$ for fitness over several generations. In our study, we addressed this question by

103 combining classical quantitative genetics with experimental evolution in the model organism Tribolium

104 castaneum. We used a two-generation half-sib/full-sib breeding design to estimate genetic variances of fitness

105 traits in four different conditions (control, dry, hot and hot-dry) representing two often co-occurring stressors,

106 heat and drought. We measured offspring number as estimate of fitness in the F1 generation, and body size in

107 the F2 generation, as an additional trait, often use as fitness proxy. We evaluated adaptation to heat, drought

108 and their combination after 20 generations of experimental evolution. Thus, our experimental setup allowed

109 us to explore many different facets of adaptation and to ask: how the adaptive potential changes under

110 stressful conditions, whether trade-offs between female and male fitness can constrain adaptation, and by

111 testing for genotype-by-environment interactions ( $\mathrm{G} \times \mathrm{E}$,) to which extent resistance to different stressors

112 shares a common genetic basis. Having obtained such estimates in the founder populations, we could then

113 gain deeper insights into the process of adaptation. Linking experimental evolution and classical quantitative

114 genetics proved to be a powerful approach to evaluate the predictive power of genetic variance for fitness,

115 and obtain a better understanding of the observed adaptation after 20 generations in new environments.

\section{Material and Methods}

\section{$118 \quad$ Animal rearing and stress treatments}

119 We used the Tribolium castaneum Cro1 strain (Milutinović et al. 2013), an outbred lab strain collected from a 120 wild population in 2010 , kept at high population size $(>10,000)$ and adapted to lab standard conditions $\left(33^{\circ} \mathrm{C}\right.$, $12170 \%$ relative humidity) for more than 30 generations. Beetles were kept in $24 \mathrm{~h}$ darkness on organic wheat 122 flour mixed with $10 \%$ organic baker's yeast. We sterilized the flour and yeast by heating them for $12 \mathrm{~h}$ at $80^{\circ} \mathrm{C}$ 123 before use. We measured fitness as well as size in control (=standard) conditions and three stress treatments 
124 with increased temperature or/and decreased humidity. The conditions in the treatments were: Hot: $37^{\circ} \mathrm{C}$ and

$12570 \%$ relative humidity, Dry: $33^{\circ} \mathrm{C}$ and $30 \%$ r. h., Hot-Dry: $37^{\circ} \mathrm{C}$ and $30 \%$ r. h.

126 In order to be able to estimate genetic variances, we applied a split-brood paternal half-sib breeding design.

127 We produced 147 half-sib families by mating virgin males to three virgin females (Figure S1A). Half- as well as

128 full-sib families were split across all conditions (Figure S1A). Male and female offspring (four females and two

129 males per full-sib family and condition) were separated at the pupal stage and transferred to $10 \mathrm{~mL}$ tubes with

$1301 \mathrm{~g}$ of medium and remained there until they were used for the fitness assay eight weeks later.

$132 \quad$ Fitness Assay and producing double first cousins

133 To estimate fitness, we mated each virgin male with two unrelated virgin females from the same condition in

$13415 \mathrm{~mL}$ tube with $1 \mathrm{~g}$ medium. The male was removed after $24 \mathrm{~h}$ and females transferred into two separate

135 tubes. Females were removed from the tubes after one week of egg laying, and 9g medium was added to

136 provide food for the developing offspring. After five weeks the number of adult offspring was counted. While

137 we conducted the matings for the fitness assay, we followed a specific crossing design and always crossed two

138 pairs of full-sib families (Figure S1B). Individuals resulting from these crosses (F2) were double first cousins.

142 Body size was measured in the F2, i.e. in the offspring of beetles that were used for the fitness assay. To 143 estimate body size, we used the centroid size of the abdominal segment IV as proxy for total size since it can 144 be measured more accurately than dry weight in very small insects and shows a high correlation with body 145 mass (Wickman and Karlsson 1989; Honěk 1993). We decided to use size of the abdominal segment because 146 we could measure it with higher accuracy than the total size, which strongly depends on whether an individual 147 is in perfectly stretched position. The size of other parts of the body show a high correlation with this segment 148 (Supplemental Figure S3). Dead beetles were fixed with a double-faced Scotch tape dorsally on a microscope 149 slide. Sex was determined based on the sex patches of males at the inside of the femur. After sexing, all legs of 
150 the beetles were removed. Slides with single specimens were placed under a Wild M8 Heerbrugg M8

151 dissection microscope with a transmitted light stand. Two further light sources from above were installed for

152 better illumination. Images were captured with a 25x magnification using a Leica DFC495 digital camera

153 connected to a PC running the Microscope imaging software LAS v4.6.2. File utility program "tpsUtil" was used

154 to build tps files from the images and tpsdig2 for setting the landmarks (see

155 http://life.bio.sunysb.edu/ee/rohlf/software.html for program information). To estimate beetle size, four

156 landmarks were set on the ventral part of the abdominal IV

157 (https://figshare.com/articles/_Morphology_of_the_red_flour_beetle_Tribolium_castaneum_/759706/1). The

158 centroid size was calculated using the free software Past 3.14 (for information see:

159 http://folk.uio.no/ohammer/past/).

160 Effects of condition on fitness and size

161 Statistical analysis was conducted in R version 3.4.2 (R Core Team 2017). We used linear mixed models as

162 implemented in the Rpackage Ime4 1.1-17 (Bates et al. 2015) to explore the effects of treatments on fitness

163 and size. For fitness analysis we included treatment and batch (time of fitness assay) as fixed effects and

164 mother identity as random effect to account for non-independence since some of our measured individuals

165 were full-sibs. For analysing size we included batch (individuals that grow up at the same time), treatment and

166 sex as well as their interaction as fixed effects and mother identity as random effect. Significance and

167 confidence intervals were obtained using the Rpackages Ismeans 2.27-62 (Lenth 2016) and Imertest 3.0-1

168 (Kuznetsova et al. 2017).

172 Adaptation

173 We used ten replicate lines per condition originating from the same ancestral population (same as before, 174 Cro1) and let them adapt for 20 generations (Supplemental Figure S2). Each new generation was set up by 175 randomly selecting 120 pupae and placing them into a new vial with $70 \mathrm{~g}$ medium. One selection line in Dry 
176 became extinct. Adult beetles of generation 20 from all selection lines were transferred to control conditions,

177 in which they stayed for one week to mate and lay eggs. After removal of the adults, we waited until their

178 offspring had reached the pupal stage and separated males and females. These individuals (generation 21)

179 developed completely in control conditions. When they had reached the adult stage, each virgin male was

180 mated with a virgin female of the same selection line and their offspring was transferred to all four conditions

181 in the egg stage, resulting in full-sib families split across all conditions (Figure S2). As soon as these offspring

182 (generation 22) had reached the pupal stage, males and females were separated. To compare fitness of

183 different selection lines and test for adaptation, a virgin male and a virgin female of the same selection line in

184 the same condition, but from different families were mated and the number of adult offspring produced within

185 four days of mating and egg laying was used as a fitness estimate. To test for adaptation, we compared

186 whether offspring number of selection lines in their native condition was significantly higher compared to non-

187 adapted control lines. First, we analysed each condition separately. We used linear mixed models including

188 selection regime as fixed effect and lines and families nested within lines as random effects using the

189 Rpackage Ime4 (Bates et al. 2015). We were further interested to test for correlated responses, i.e. whether

190 adaptation to a certain stress treatment could increase fitness in another. For this we run one analysis using

191 the complete data set. Selection regime, conditions, and their interaction were used as fixed effects, lines,

192 families nested within lines, and line-treatment interaction as random effects. Reported effect sizes and

193 standard errors were obtained from the summary output of the model. $p$-values and confidence intervals were

194 computed with the Rpackages Imertest (Kuznetsova et al. 2017) and Ismeans (Lenth 2016). Statistical analyses

195 were conducted in R (R Core Team 2017). Model diagnostic plots are shown in supplemental Figure S6 and the

196 complete results in Table S2.

\section{Quantitative genetic analyses}

199 We estimated genetic variances, covariances and correlations using an animal model and restricted maximum

200 likelihood estimation as implemented in Asreml version 3.0 (Gilmour et al. 2009). The animal model is a linear 201 mixed effect model that uses all known relationships from a pedigree as random effect to partition observed 
202 variance into additive genetic variance and other sources of variance (Kruuk 2004). Non-additive genetic

203 relationship matrices were created with the Rpackage nadiv 2.16.0.0 (Wolak 2012). All models were run in R by

204 Asreml-R (Butler et al. 2009). All models reached convergence.

207 First, we ran a series of univariate models for each condition separately with offspring number as response 208 variable, batch (samples where fitness assay was started on the same day) as fixed effect, additive genetic 209 effects of females and additive genetic effects of males as random effects. Maternal identity (mother of 210 female) was included as random effect to account for resemblance of full-sibs due to maternal, common 211 environment or non-additive genetic effects. Significance of random effects was determined by likelihood ratio 212 tests, testing whether excluding a certain random effect resulted in a significantly worse model. Reported P213 values are one-tailed since we tested whether a certain variance is different from zero and variances cannot 214 become negative (Wilson et al. 2010). Although count data, it can be analysed assuming a Gaussian 215 distribution when distribution converges towards a normal distribution (de Villemereuil 2018). Distribution of 216 offspring numbers are shown in Figure S4. Model diagnostic plots and more details are given in the 217 Supplement (Figure S5). For estimating cross-sex genetic correlation we used the same univariate models but 218 included a covariance between female and male additive genetic effects. Maternal effects were removed in 219 subsequent analyses since they were very small and non-significant. Studies so far used bivariate models to 220 investigate cross-sex genetic correlations (Brommer et al. 2007; Foerster et al. 2007; McFarlane et al. 2014;

221 Punzalan et al. 2014; Wolak et al. 2018). In case of fitness this could be problematic, because the same 222 observation (number of adult offspring resulting from a mating) would be used twice. Using univariate models 223 including additive genetic effects of females and males avoids pseudoreplication. Significance of genetic 224 correlations was determined by two-tailed likelihood ratio tests comparing a model including correlations to a 225 model with correlations set to zero. In order to test for genotype-by-sex interaction (G x S), which indicates 226 that a genotype is differently expressed in males and females, we compared the model to a constrained model 
227 with genetic correlation fixed to one. To examine whether additive genetic effects of females and males were

228 different, we tested whether the unconstrained model was significantly better than a model with $\mathrm{V}_{\mathrm{A}}$ of females

229 and $V_{A}$ of males forced to be equal within each condition.

230 For estimating cross-condition genetic covariances and correlations we used bivariate models with fitness in

231 two conditions as response, batch as fixed and additive genetic effects of females and males as random effects.

232 Since each individual was only measured in one condition, residual covariance was set to zero. Significance of

233 correlation was assessed by comparing to a model with correlation fixed to zero using two-tailed likelihood

234 ratio tests. Genetic correlations between traits measured in different environments that are significantly less

235 than unity, indicate a G x E interactions (Kruuk 2004; Charmantier and Garant 2005). Comparison with a model

236 with correlation fixed to one was used to test for $\mathrm{G} \times \mathrm{E}$ (Wilson et al. 2010). In all bivariate models, covariance

237 between male effects was set to zero, because male effects were very small and not significant in some

238 conditions and hence it was not meaningful to estimate covariances.

239 To allow comparisons across different conditions, we calculated $\mathrm{h}^{2}$ and $\mathrm{CV}_{\mathrm{A}}\left(\mathrm{CV}_{\mathrm{A}}\right.$ is the square root of $\mathrm{V}_{\mathrm{A}}$ divided

240 by the phenotypic mean of the trait, multiplied by 100) of fitness for males and females in each condition. $I_{A}\left(V_{A}\right.$

241 divided by trait mean squared, multiplied by 100 (Houle 1992)) was calculated as an estimate for the

242 proportional change after one generation. Standard errors of $\mathrm{CV}_{\mathrm{A}}$ and $\mathrm{I}_{\mathrm{A}}$ were computed as described in Garcia-

243 Gonzalez et al., (2012).

244 To assess whether genetic variances were significantly affected by environmental change, we used a 245 multivariate model with fitness in each condition as separate response variable. Since fitness between 246 conditions differed in mean and variance, we standardized data. We applied two different standardizations: 1) 247 mean standardized (i.e. relative fitness) and 2) dividing by the standard deviation (sd) to have a variance of 248 one. We then tested whether constraining the multivariate model and forcing $V_{A}$ of standardized fitness to be 249 equal in all conditions resulted in a significantly worse model. If the constrained models are worse, we can 250 conclude that mean standardized $V_{A}\left(I_{A}\right.$ of absolute numbers), or sd standardized $V_{A}\left(h^{2}\right.$ of absolute numbers) 251 are significantly influenced by the environment. Similarly, we tested whether genetic cross-condition 252 correlations were different. We used a multivariate model and constrained all pairwise cross-condition 
253 correlations to be equal. We then tested if this model was significantly worse than an unconstrained model

254 using a two-tailed likelihood-ratio test.

257 Our crossing design during the fitness assay (reciprocal crossing of two pairs of full-sibs, see Figure S2) allowed 258 us to estimate non-additive genetic variance $\left(V_{D}\right)$ in the following generation ( $F 2$ ). In a paternal half-sib 259 breeding design these effects contribute to resemblance among full-sibs and cannot be separated from 260 maternal or common environmental effects. In contrast, double first cousins (DFC) share non-additive genetic 261 effects without being confounded by maternal effects or a common environment. We partitioned the 262 observed variance for size into additive $\left(V_{A}\right)$, maternal $\left(V_{M}\right)$, non-additive $\left(V_{D}\right)$, and residual variance $V_{R}$ while 263 controlling for batch effects. Batches represent individuals that grew up at the same time, and thus accounts 264 for variations in the medium or lab temperature. Similar to the analysis of fitness data, we first analysed each 265 condition separately using univariate animal models with batch as fixed and maternal, additive, and non266 additive genetic effects as random effects. To get sex specific estimates, we analysed male and female size 267 separately. Genetic correlations between sexes and between conditions were assessed by bivariate models. 268 Significance of correlations, $\mathrm{G} \times \mathrm{E}$, environmental effects on $\mathrm{I}_{\mathrm{A}}$ and $\mathrm{h}^{2}$ of size were tested in the same way as we 269 described before.

271 To demonstrate how responses are affected by covariances between the sexes we used estimated variances 272 and covariances between male and female additive genetic effects of relative fitness to predict fitness increase

273 after one generation. We applied the multivariate breeder's equation $\Delta z=G \beta$ (Lande 1979), where $\Delta z$ is a 274 vector of changes in the trait means, $G$ the $2 \times 2$ genetic variance-covariance matrix for female and male 275 relative fitness estimated in each condition, and $\beta$ a vector of selection gradients. We used a vector of 276 selection gradients that assumed equal selection on male and female fitness, $\beta^{\top}=[1,1]$. Total change in fitness is 277 then the sum of fitness increase in females and in males. Alternatively, we may consider male additive genetic 278 effects as indirect genetic effects on female reproductive output. Joint effects of direct and indirect selection 
on evolutionary changes of a single trait can be calculated as described in Bijma \& Wade (2008) (equation 14):

$\sigma_{T}^{2}=\sigma_{A_{D}}^{2}+2(n-1) \sigma_{A_{D I}}^{2}+(n-1)^{2} \sigma_{A_{I}}^{2}$, where $\sigma_{T}^{2}$ is the total heritable variance, $\sigma_{A_{D}}^{2}$ additive genetic variance

281 for direct effects (female $\mathrm{V}_{\mathrm{A}}$ ), $\sigma_{A_{I}}^{2}$ additive genetic variance for indirect effects (male $\mathrm{V}_{\mathrm{A}}$ ), and $n$ gives the

282 number of interacting individuals (here, it equals two). In case of relative fitness, this total heritable variance is

283 equal to the predicted relative increase in fitness (Fisher 1930). Thus, considering male and female fitness as

284 two separate traits and applying the multivariate breeder's equation, or treating additive genetic effects of

285 males as indirect genetic effects on female fitness is equivalent.

\section{$287 \quad$ Results}

\section{$288 \quad$ Effect of treatment}

289 It was previously shown that all treatments had a highly significant effect on offspring number (Koch and

290 Guillaume 2020) with the effect of heat being stronger and the lowest offspring number when heat and

291 drought were combined. (Figure 1A). Treatments also showed a significant effect on size $\left(F_{3,7710}=76.30, p<\right.$ 292 2.20E-16, Table S1) and size decreased in the stressful conditions. Similar to offspring number, the effect of 293 drought was smaller. Combining both stressors did not result in an additional decrease in size. In contrast, we 294 observed the lowest size in Hot and higher sizes in Hot-Dry (Figure 2A). The difference between males and 295 females was significant $\left(F_{1,7759}=518.19, p<2.20 E-16\right)$ with females being larger. We also detected a significant 296 sex-by-condition interaction $\left(F_{3,7759}=7.27, p=8.16 \mathrm{E}-04\right)$ indicating that males were more sensitive to stressful 297 conditions (Figure 2A). 
A

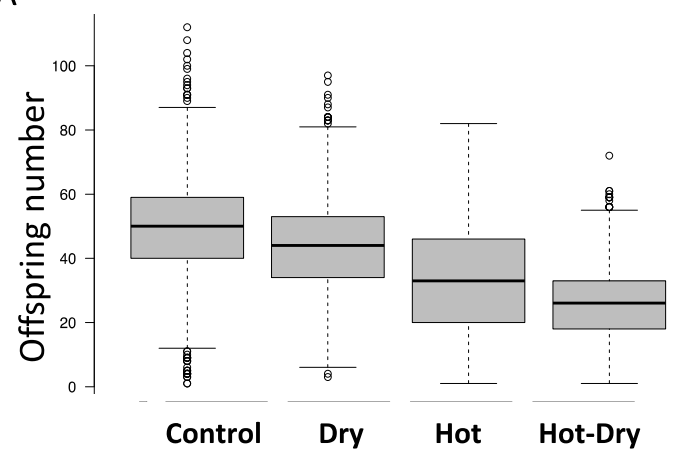

C

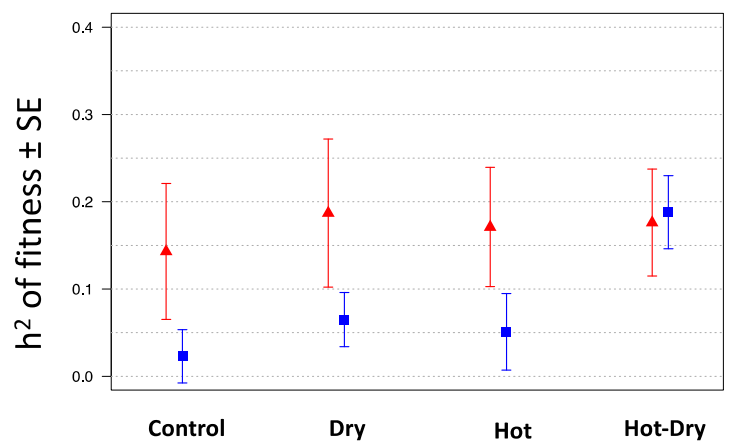

B

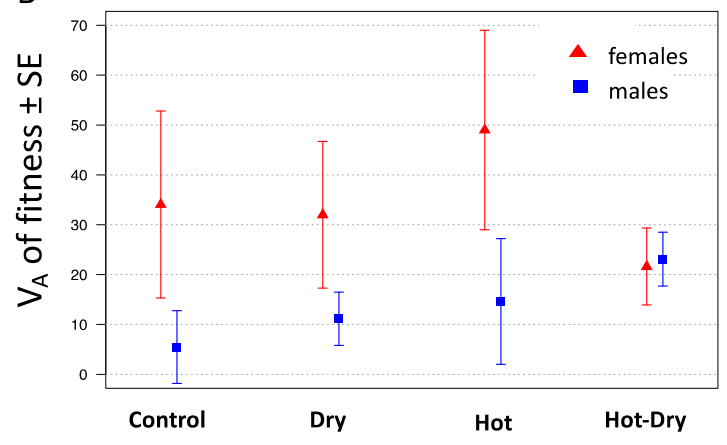

D

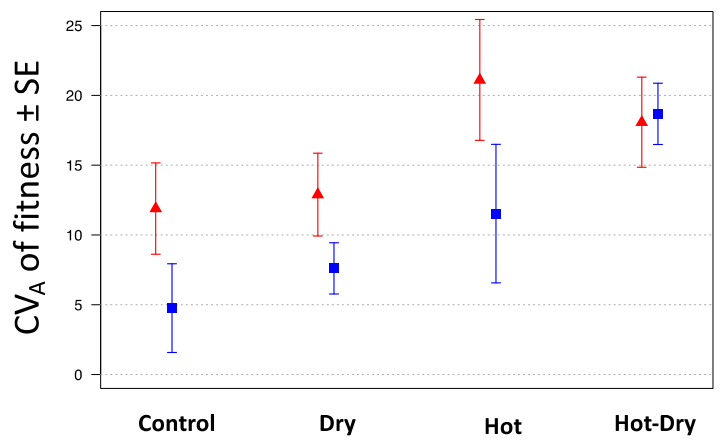

Figure 1: Fitness (= number of adult offspring) $(A)$ and estimates for its additive genetic variance $\left(V_{A}\right)(B)$, heritability $\left(h^{2}\right)$

300 (C), and coefficient of $V_{A}\left(\mathrm{CV}_{A}\right)$, which is the square root of $\mathrm{V}_{\mathrm{A}}$ divided by the phenotypic trait mean, multiplied by 100 (D) in

301 female and male flour beetles (Tribolium castaneum) in four different environmental conditions. 

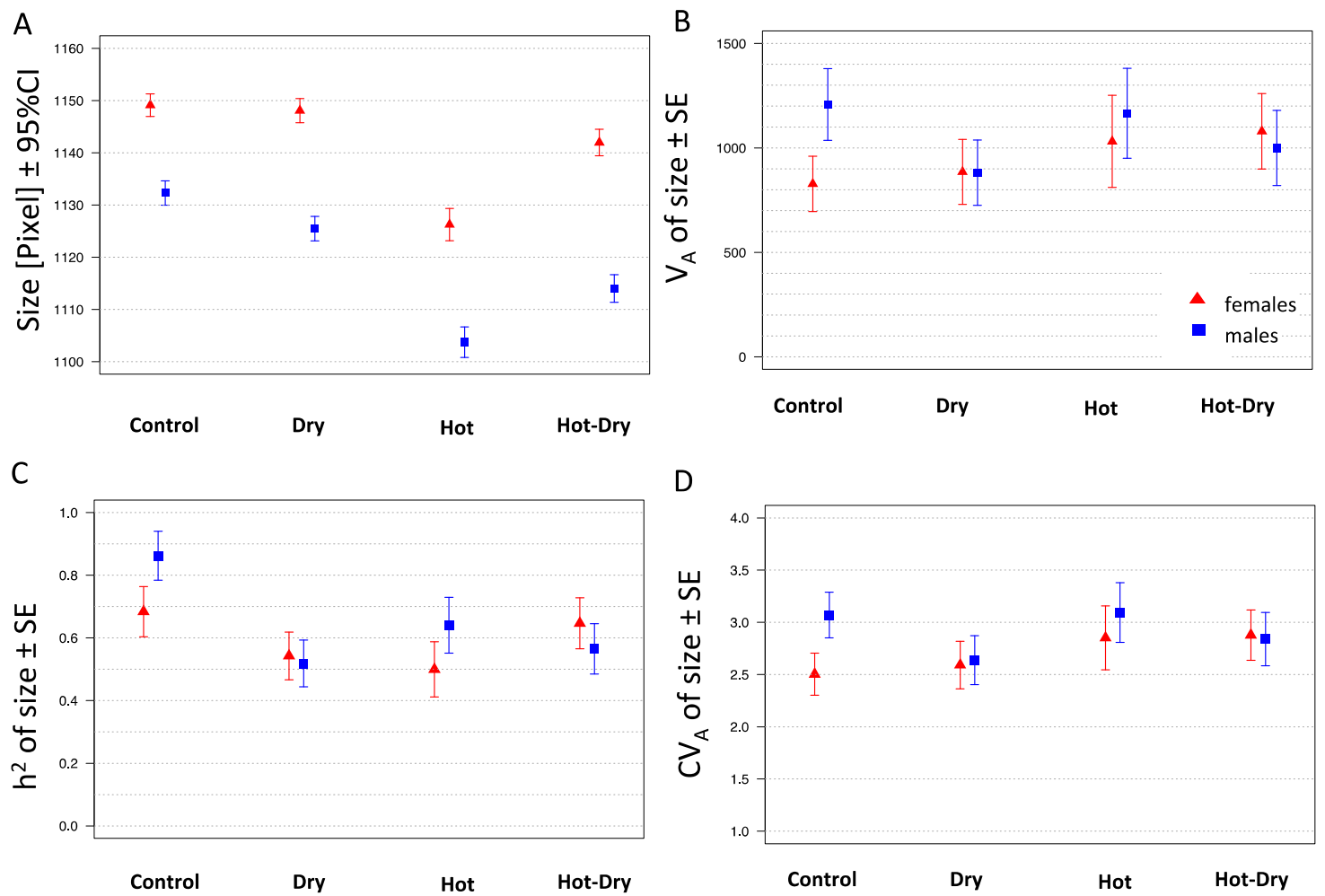

Figure 2: Body size (centroid size of abdominal segment IV) (A) and estimates for its additive genetic variance $\left(\mathrm{V}_{\mathrm{A}}\right)(\mathrm{B})$, heritability $\left(\mathrm{h}^{2}\right)(\mathrm{C})$, and coefficient of $\mathrm{V}_{\mathrm{A}}\left(\mathrm{CV}_{\mathrm{A}}\right)(\mathrm{D})$ in female and male flour beetles (Tribolium castaneum) in four different environmental conditions.

\section{$308 \quad$ Fitness}

$309 \mathrm{~V}_{\mathrm{M}}$, which consisted of variance due to maternal effects, common environment and non-additive effects, was 310 not significant in any condition. We found significant $V_{A}$ for female fitness in all conditions (Table 1). $V_{A}$ for 311 males was generally smaller $(P=8.10 \mathrm{E}-03)$ and not significantly different from zero in Control and Hot. A 312 remarkable exception was Hot-Dry, where male $V_{A}$ was increased and of the same magnitude as female $V_{A}$

313 (Figure 3). $\mathrm{V}_{\mathrm{A}}$ in females was similar in Control and Dry but increased in Hot and was reduced in Hot-Dry (Figure 314 1B). Since residual variances changed simultaneously with $V_{A}$ (Table 1), $h^{2}$ for females was similar in all stress 
$316=2.75 \mathrm{E}-03)$, but not females $(\mathrm{P}=0.55)$ to differ significantly between conditions. Similarly, $\mathrm{I}_{\mathrm{A}}$ was affected by 317 conditions in males $(P=8.87 E-05)$, but not in females $(P=0.07)$. When we tested a model constraining $V_{A}$ of 318 females and males to be the same in all conditions, we found it to be significantly worse $(P=2.46 \mathrm{E}-08)$ than an 319 unconstrained model, thus showing that the total amount of heritable genetic variance $\left(V_{A}\right.$ of females and 320 males) was different between treatments.

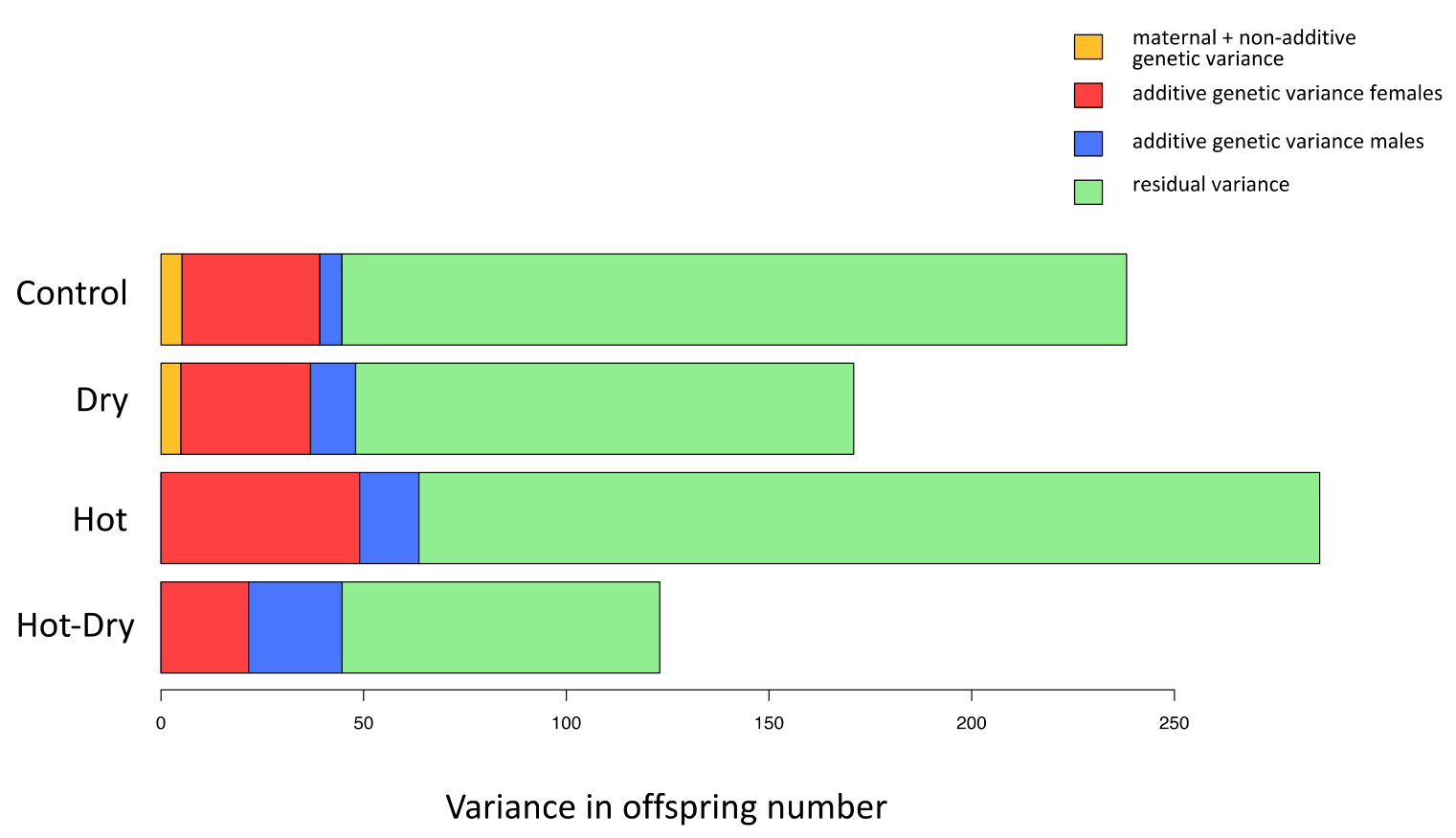

321 Figure 3: Variance components of offspring number of Tribolium castaneum in four different environmental conditions 322 estimated using univariate animal models. 
Table 1: Genetic variances $\left(V_{A}\right.$ : additive genetic variance; $V_{M}$ : maternal variance; $V_{R}$ : residual variance), heritability $\left(h^{2}\right)$, coefficient of additive genetic variance $\left(\mathrm{CV}_{\mathrm{A}}\right)$ of offspring number and cross-sex additive genetic covariances $\left(\mathrm{COV}_{\mathrm{A}}\right)$ and correlations $\left(r_{A}\right)$ in different environmental conditions. Estimates for genetic variances were obtained from a univariate animal model including additive genetic effects of males and females as random effects. For $\operatorname{COV}_{\mathrm{A}}$ and $\mathrm{r}_{\mathrm{A}}$ univariate animal models were used with covariance between female and additive genetic effects. $\mathrm{N}$ gives the number of reproducing females used for the analysis. All significant results are in bold.

\begin{tabular}{|c|c|c|c|c|}
\hline & \multicolumn{4}{|c|}{ Condition } \\
\hline & Control & Dry & Hot & Hot-Dry \\
\hline $\mathbf{N}$ & 1514 & 1603 & 1005 & 1396 \\
\hline mean (SE) & $49.09(0.4)$ & $43.88(0.36)$ & $33.18(0.55)$ & $25.73(0.31)$ \\
\hline$V_{A}$ females (SE) & $34.06(18.75)$ & $32.00(14.71)$ & $48.99(20.02)$ & $21.62(7.71)$ \\
\hline$h^{2}$ females (SE) & $0.14(0.08)$ & $0.19(0.09)$ & $0.17(0.07)$ & $0.18(0.06)$ \\
\hline $\mathrm{CV}_{\mathrm{A}}$ females (SE) & $11.89(3.27)$ & $12.89(2.97)$ & $21.10(4.32)$ & $18.07(3.23)$ \\
\hline $\mathrm{V}_{\mathrm{A}}$ males $(\mathrm{SE})$ & $5.44(7.29)$ & $11.14(5.35)$ & $14.62(12.59)$ & $23.09(5.40)$ \\
\hline$h^{2}$ male (SE) & $0.02(0.03)$ & $0.07(0.03)$ & $0.05(0.04)$ & $0.19(0.04)$ \\
\hline $\mathrm{CV}_{\mathrm{A}}$ males (SE) & $4.75(3.18)$ & $7.61(1.83)$ & $11.53(4.97)$ & $18.68(2.20)$ \\
\hline $\mathrm{V}_{\mathrm{M}}$ (SE) & $5.18(10.30)$ & $4.90(7.55)$ & 2.26E-07 (5.03E-0.5) & $2.76 \mathrm{E}-05(2.21 \mathrm{E}-06)$ \\
\hline $\mathrm{V}_{\mathrm{R}}$ (SE) & $193.39(13.65)$ & $122.88(9.79)$ & $222.27(20.00)$ & 78.35 (6.27) \\
\hline$V_{P}$ (SE) & $238.22(8.88)$ & $170.91(6.33)$ & $235.88(13.10)$ & $123.05(4.96)$ \\
\hline $\mathrm{COV}_{\mathrm{A} \text { female,male }}(\mathrm{SE})$ & $25.73(6.29)$ & $11.75(4.92)$ & $-9.24(11.02)$ & $11.54(4.21)$ \\
\hline$r_{A \text { female, male }}$ (SE) & $1.55(0.66)$ & $0.54(0.22)$ & $-0.36(0.45)$ & $0.52(0.18)$ \\
\hline
\end{tabular}

Genetic correlations between fitness in different conditions were always positive (Figure 4) and significant. However, we found significant differences between the different cross-condition correlations $(P=0.05)$. Correlations were slightly lower when the conditions differed in temperature and humidity (Control-Hot-Dry, Dry-Hot) (Figure 4). We found significant G x E between Control and Dry, Control and Hot-Dry as well as Dry and Hot. 


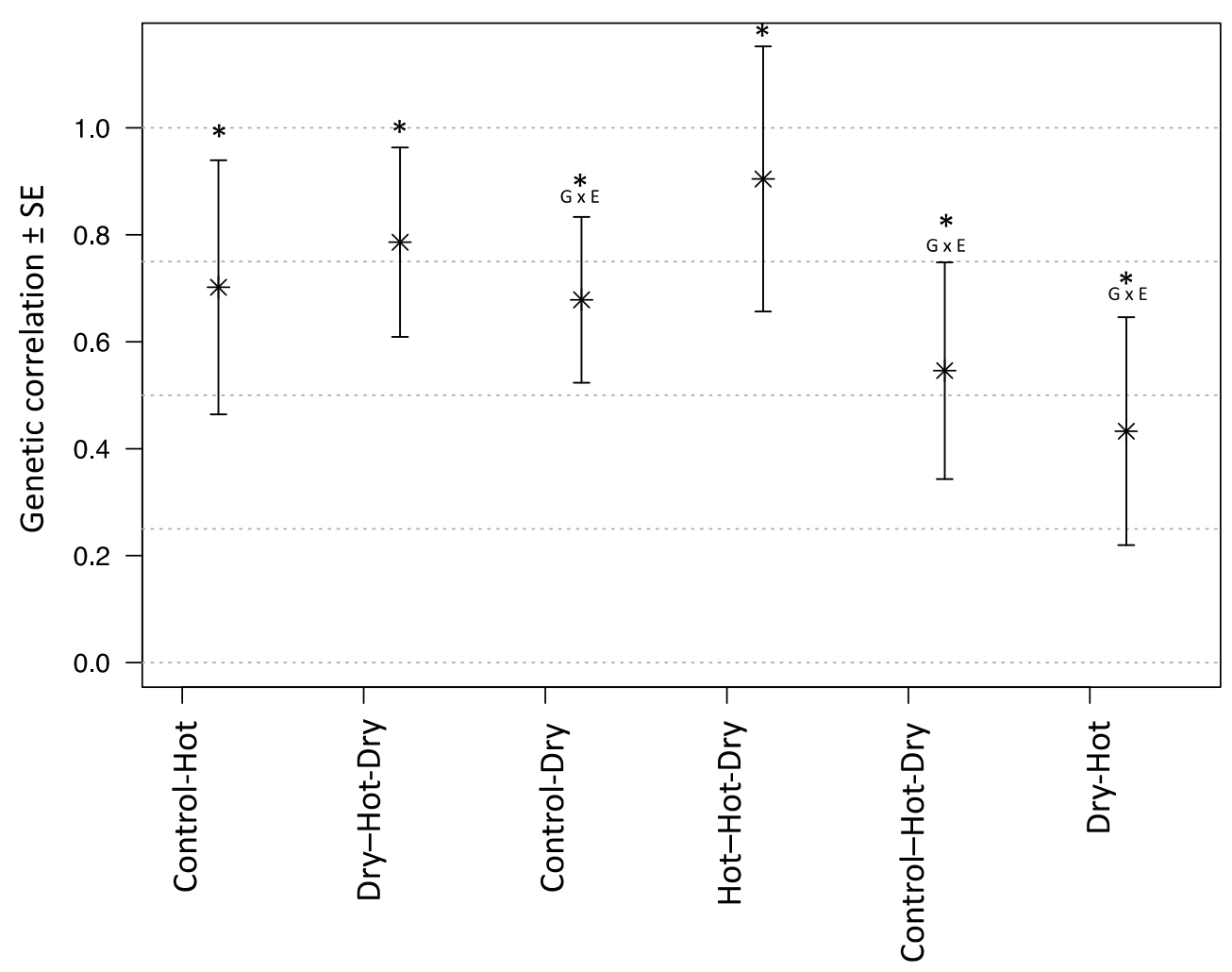

Figure 4: Pairwise cross-environment genetic correlations of fitness in Tribolium castaneum estimated using bivariate animal models. * show significant correlations, i.e. significantly different from zero. G x E (= genotype by environment interactions) indicate correlations significantly different from one. Control: $33^{\circ} \mathrm{C}, 70 \%$ relative humidity (r.h.); Dry: $33^{\circ} \mathrm{C}$, $30 \%$ r.h.; Hot: $37^{\circ} \mathrm{C}, 70 \%$ r.h.; Hot-Dry: $37^{\circ} \mathrm{C}, 30 \%$ r.h.

343 Genetic correlations in fitness between females and males (Figure 5) were significant in Control $(P=1.02 \mathrm{E}-06)$,

344 Dry $(P=1.34 \mathrm{E}-02)$, and Hot-Dry $(P=4.47 \mathrm{E}-04)$, but not in Hot $(P=0.10)$. The correlation was highest in Control

345 (Table 1, Figure 5). It decreased in the stress treatments and became even negative in Hot. In all conditions 346 except Control, we found that the correlation was significantly different from one (Dry: $\mathrm{P}=1.95 \mathrm{E}-03$; Hot: $\mathrm{P}=$ 347 5.78E-03; Hot-Dry: $P=1.26 \mathrm{E}-04)$, indicating $\mathrm{G} \times \mathrm{S}$, i.e. that genetic basis of fitness is different in the sexes. $\mathrm{I}_{\mathrm{A}}$, 348 the expected evolutionary change as percentage of the mean, was highest in Hot and smallest in Control 

with the largest relative fitness increase in Hot. However, given the negative correlation between male and increase of mean fitness of $3.90 \%$ in Dry, $3.89 \%$ in Hot, and $10.20 \%$ in Hot-Dry per generation. Assuming $V_{A}$

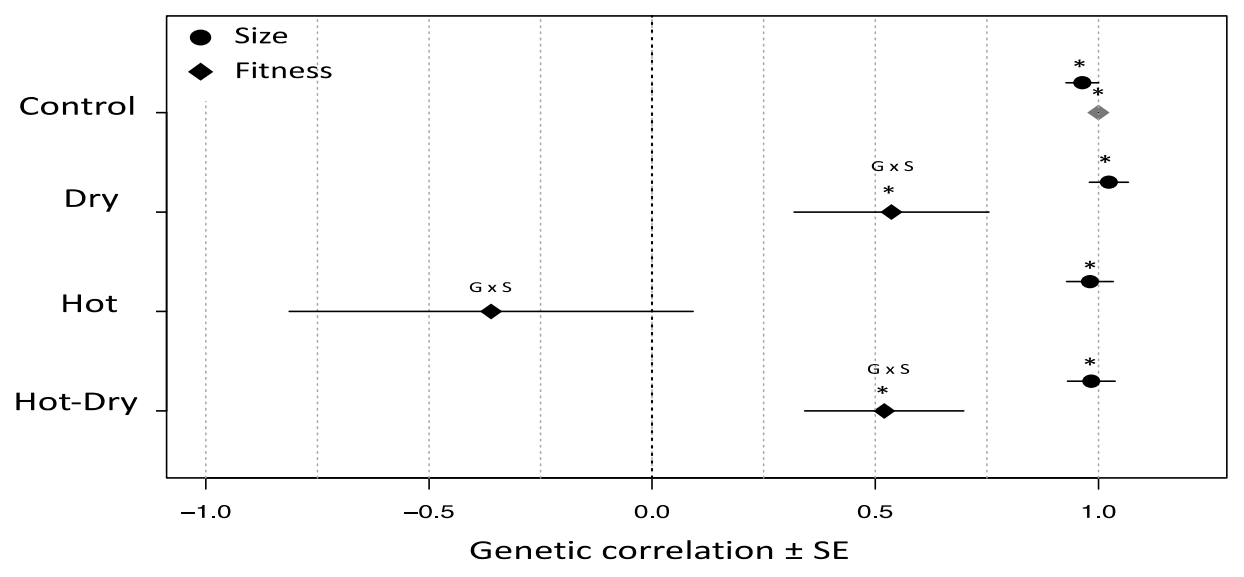

Figure 5: Cross-sex genetic correlations for size and fitness in Tribolium castaneum under different environmental conditions. ${ }^{*}$ indicates a significant correlation. G x S (= genotype by sex interaction) indicates that correlation is significantly different from one. Estimate for genetic correlation of fitness in Control was bounded at one and no SE was available. Using models with unconstrained variances and covariances yielded an estimate of $1.55 \pm 0.66$. $V_{A}$ of males was small in Control, which impedes precise estimation of cross-sex genetic correlation.

$V_{A}$ in size was highly significant in both sexes and in all conditions $(P<0.001)$. The results for $V_{D}$ were less clear. 
366 non-additive effects contributed little to observed variation (Figure 6). In Dry and Hot, $V_{D}$ was not significant,

367 but variance estimates were high when it was included in the model (Table 2, Figure 6). A model without non-

368 additive effects resulted in much higher estimates of $\mathrm{V}_{\mathrm{A}}$ in Dry and Hot (Supplemental Table S3). In most cases,

369 P-values for $V_{D}$ were far from significance $(P>0.2)$, but for females in Dry and in Hot $P$-values were lower

370 although still not significant $(P=0.13$ and $P=0.10)$. When we combined males and females and added sex as

371 fixed effect in the model, we obtained a P-value of 0.075 in Hot. Genetic correlations for size across conditions

372 were always positive (Supplemental Figure S7), but no clear pattern emerged. We found significant G x E in

373 female size between Control-Dry $(P=2.78 E-03)$. In male size, $G \times E$ was significant between Control-Dry $(P=$

374 6.39E-03), Dry-Hot $(P=5.39 E-03)$, Dry-Hot-Dry $(P=0.04)$, and Hot-Hot-Dry $(P=3.93 E-03)$. Genetic correlations

375 between female and male size were close to one (Table 2, Figure 5) in all conditions, suggesting that size

376 cannot evolve independently in both sexes. $\mathrm{I}_{\mathrm{A}}$ of female and male size was not significantly different $(P=0.08)$

377 considering all conditions. Although differences in Control seemed to be substantial (Figure 4B-D), they were

378 not significant $\left(h^{2}: P=0.26 ; I_{A}: P=0.06\right)$. We found that environmental change did not influence $I_{A}$ and $h^{2}$ of

379 female size $(P=0.13 ; P=0.14)$ nor male $I_{A}(P=0.13)$, but had a significant effect on male $h^{2}(P=0.02)$. 
A

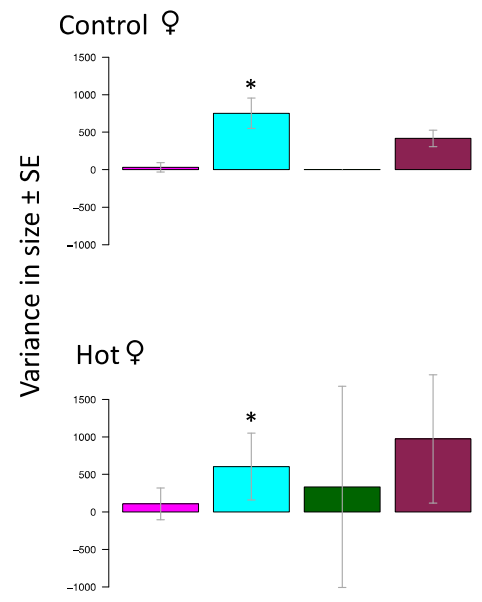

B

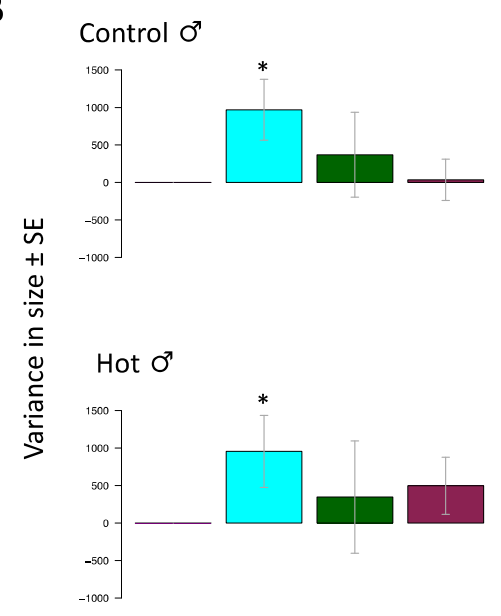

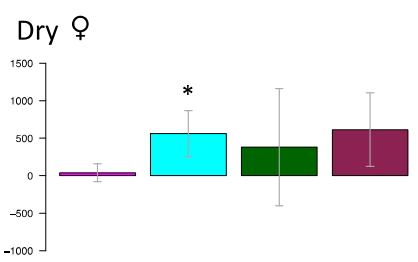

Hot-Dry P
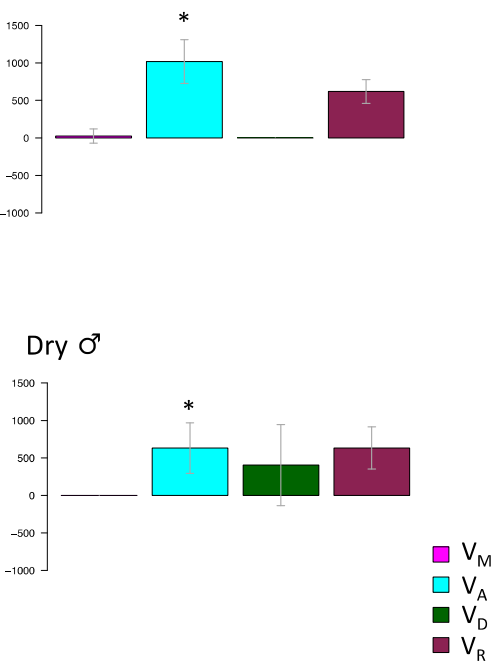

Hot-Dry ơ

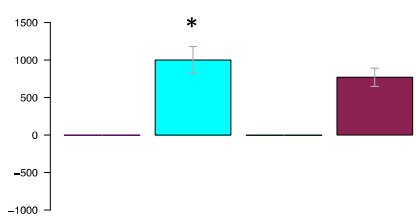

381 Figure 6: Variance components of size in female (A) and male (B) flour beetles (Tribolium castaneum) under four 382 environmental conditions: $V_{M}$ : maternal variance, $V_{A}$ : additive genetic variance, $V_{D}$ : non-additive genetic variance, $V_{R}$ :

383 residual variance. * indicates a significant variance component. 
Table 2: Body size genetic variances $\left(\mathrm{V}_{\mathrm{A}}\right.$ : additive genetic variance; $\mathrm{V}_{\mathrm{D}}$ : dominance variance; $\mathrm{V}_{\mathrm{M}}$ : maternal variance; $\mathrm{V}_{\mathrm{R}}$ : residual variance), heritability $\left(\mathrm{h}^{2}\right)$, coefficient of additive genetic variance $\left(\mathrm{CV}_{\mathrm{A}}\right)$ in female and male flour beetles and crosssex additive genetic covariances $\left(\operatorname{COV}_{A}\right)$ and correlations $\left(r_{A}\right)$ in different environmental conditions. Estimates for genetic

\begin{tabular}{|c|c|c|c|c|c|c|c|c|}
\hline & \multicolumn{8}{|c|}{ Condition } \\
\hline & \multicolumn{2}{|c|}{ Control } & \multicolumn{2}{|c|}{ Dry } & \multicolumn{2}{|c|}{ Hot } & \multicolumn{2}{|c|}{ Hot-Dry } \\
\hline Females & \multicolumn{2}{|c|}{1020} & \multicolumn{2}{|c|}{1181} & \multicolumn{2}{|c|}{837} & \multicolumn{2}{|c|}{960} \\
\hline mean (SE) & 1149.13 & $(1.10)$ & 1148.08 & $(1.18)$ & 1126.25 & $(1.57)$ & 1141.96 & (1.29) \\
\hline $\mathrm{V}_{\mathrm{A}}(\mathrm{SE})$ & 751.79 & $(201.63)$ & 560.98 & $(305.17)$ & 603.78 & $(446.35)$ & 1017.45 & (291.52) \\
\hline$V_{D}(S E)$ & $6.63 \mathrm{E}-04$ & (1.73E-04) & 379.526 & (782.07) & 333.554 & $(1342.287)$ & 8.670E-04 & $(2.21 \mathrm{E}-04)$ \\
\hline $\mathrm{V}_{\mathrm{M}}(\mathrm{SE})$ & 29.61 & (63.13) & 38.57 & (119.09) & 107.14 & $(210.86)$ & 25.58 & (96.18) \\
\hline$V_{R}(S E)$ & 417.11 & $(108.70)$ & 613.14 & $(490.95)$ & 973.23 & (854.49) & 618.81 & (157.41) \\
\hline$V_{P}(S E)$ & 1198.52 & $(72.27)$ & 1592.19 & (82.03) & 2017.74 & (117.05) & 1661.84 & $(100.31)$ \\
\hline$h^{2}$ (SE) & 0.63 & $(0.14)$ & 0.35 & $(0.18)$ & 0.30 & $(0.21)$ & 0.61 & $(0.15$ \\
\hline $\mathrm{CV}_{\mathrm{A}}$ (SE) & 2.39 & $(0.32)$ & 2.06 & $(0.56)$ & 2.18 & $(0.81)$ & 2.79 & $(0.40)$ \\
\hline Males & \multicolumn{2}{|c|}{1008} & \multicolumn{2}{|c|}{1183} & \multicolumn{2}{|c|}{834} & \multicolumn{2}{|c|}{962} \\
\hline mean (SE) & 1132.31 & (1.18) & 1125.47 & (1.19) & 1103.73 & $(1.50)$ & 1114.01 & (1.34) \\
\hline $\mathrm{V}_{\mathrm{A}}$ (SE) & 969.79 & $(406.67)$ & 632.66 & $(335.68)$ & 954.83 & $(479.70)$ & 999.70 & (179.66) \\
\hline$V_{D}(S E)$ & 369.18 & (567.38) & 404.65 & (539.19) & 346.49 & (748.68) & $4.94 \mathrm{E}-03$ & (7.90E-04) \\
\hline $\mathrm{V}_{\mathrm{M}}$ (SE) & $6.59 \mathrm{E}-06$ & (5.07E-05) & $4.88 \mathrm{E}-04$ & $(2.18 \mathrm{E}-04)$ & $6.54 \mathrm{E}-04$ & (5.00E-04) & $3.14 \mathrm{E}-04$ & $(5.02 \mathrm{E}-05)$ \\
\hline $\mathrm{V}_{\mathrm{R}}(\mathrm{SE})$ & 35.85 & $(275.83)$ & 634.64 & $(282.89)$ & 496.90 & (380.49) & 769.42 & $(123.08)$ \\
\hline$V_{P}(S E)$ & 1374.81 & $(293.93)$ & 1672.04 & (87.39) & 798.22 & (118.96) & 1769 & (98.50) \\
\hline$h^{2}$ (SE) & 0.71 & $(0.25)$ & 0.38 & $(0.19)$ & 0.53 & $(0.25)$ & 0.57 & $(0.08)$ \\
\hline $\mathrm{CV}_{\mathrm{A}}$ (SE) & 2.75 & $(0.58)$ & 2.23 & $(0.59)$ & 2.80 & $(0.70)$ & 2.84 & $(0.26)$ \\
\hline $\mathrm{COV}_{\mathrm{A} \text { female,male }}(\mathrm{SE})$ & 955.25 & $(120.93)$ & 1002.06 & $(128.10)$ & 1140.11 & (175.33) & 976.20 & $(137.10)$ \\
\hline $\mathbf{r}_{\mathrm{A} \text { female, male }}(\mathrm{SE})$ & 0.96 & $(0.04)$ & 1.02 & $(0.04)$ & 0.98 & $(0.05)$ & 0.98 & $(0.05)$ \\
\hline
\end{tabular}




\section{Adaptation after 20 generations and asymmetric correlated responses}

396 We found significant adaptation to all conditions after 20 generations, shown by significant effects of selection regime on offspring number (Dry: $F_{1,16}=10.43, p=0.005$; Hot: $F_{1,18}=4.80, p=0.042$; Hot-Dry: $F_{1,18}=14.78, p=$ 0.001, Table S2). In all treatments, the native selection lines produced significantly more offspring than nonadapted control lines (Figure 7). The largest difference and most significant fitness increase was observed in the most stressful condition Hot-Dry. In contrast to the three stress treatments, we did not find any differences between lines from different selection regimes in the ancestral control condition (Figure 7), showing that adaptation to stress treatments did not come at a cost of reduced fitness in control conditions. Interestingly, drought selection resulted in higher heat resistance. Dry lines showed a significantly higher offspring number in Hot and Hot-Dry (estimated increase in offspring number relative to Control lines: $12.10 \pm 2.23, p=3.64 E-07$ in Hot; $12.18 \pm 2.18, p=4.20 \mathrm{E}-07$ in Hot-Dry ). In Hot, they performed even better than the native Hot-lines (mean offspring number in Hot [95\% Cl]: Control-lines: 24.59 [20.71, 28.47]; Hot-lines: 29.94 [26.11, 33.77]; Dry-lines: 35.69 [31.77, 39.61]; Hot-Dry-lines: 36.91 [33.14, 40.68]). We did not observe such a correlated response in the Hot-lines (Figure 7). Their offspring number in Dry was not different from those of ControlLines. Lines in Hot-Dry that adapted to a combination of heat and drought showed an increased fitness in both single stressor treatments (in Dry: $4.45 \pm 2.10, p=0.04$; in Hot: $11.66 \pm 2.17, p=1.20 \mathrm{E}-04$ ). Despite high genetic correlation in fitness between Hot and Hot-Dry estimated in the first generation, we found no correlated response of Hot-lines to Hot-Dry conditions. Their offspring number was not different from nonadapted Control-Lines (Hot-lines: 23.14 [19.43, 26.84 CI]; Control-lines: 23.03 [19.30, $26.76 \mathrm{CI}]$ ). 


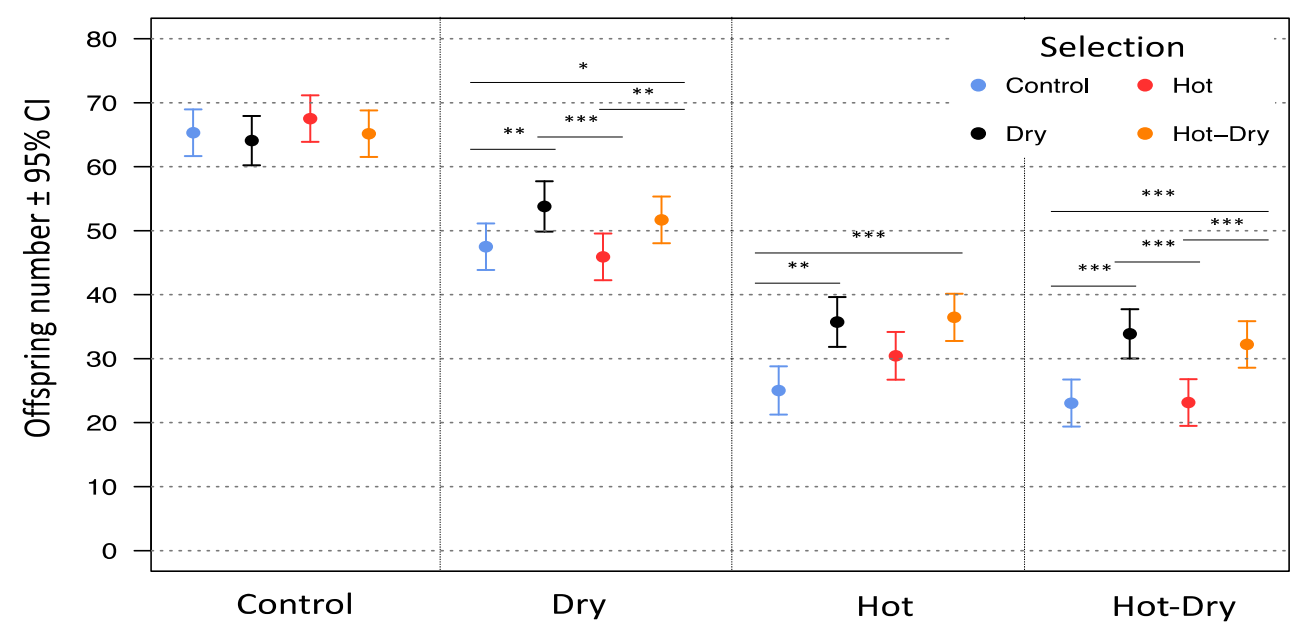

$416 \quad$ Figure 7: Mean offspring number per female of lines from different selection regimes under different environmental

417 conditions. Significant differences between selection regimes within the same condition (Tukey's HSD post hoc test, $\mathrm{p}$ 418 values adjusted for multiple comparisons) are indicated by ${ }^{*} \mathrm{P}<0.05,{ }^{* *} \mathrm{P}<0.01,{ }^{* * *} \mathrm{P}<0.001$

420 The observed changes in fitness after experimental evolution were much lower than the predicted total 421 changes. Observed fitness increases relative to Control-lines were: Dry: $16.43 \pm 3.24 \%$, Hot: $22.37 \pm 4.34 \%$ 422 and $46.29 \pm 2.17 \%$ in Hot-Dry. Nonetheless, in agreement with our predictions we observed the strongest 423 increase in Hot-Dry and similar increases in Dry and Hot. 


\section{Discussion}

431 Consequences of environmental change on persistence of populations strongly depend on their ability to 432 adapt either by plastic or genetic changes. The stress conditions we applied, had a strong impact on fitness.

433 Offspring number in Hot-Dry was reduced to ca. $50 \%$ of control level thus clearly showing that plasticity was

434 not able to fully compensate for the negative effects of environmental change. We found significant $V_{A}$ in

435 fitness in all conditions indicating potential to adapt by genetic changes with no evidence that environmental

436 changes lowered the adaptive potential. In contrary, $I_{A}$ of female and male fitness increased in the stress

437 treatments, which should facilitate adaptation. Accordingly, we found significant adaptation to all conditions

438 after 20 generations of experimental evolution. We also did not find strong evidence of genetic constraints on

439 adaptation between stressors or sexes as most genetic correlations were positive (but not in Hot, see Figure 5).

440 Taking account of both male and female variances and covariance in fitness allowed us to make better

441 predictions of fitness increase. All in all, our study shows that although it is not possible to predict the precise

442 fitness increase over this period of 20 generations, we can make qualitative predictions about adaptation

443 based on $\mathrm{V}_{\mathrm{A}}$ estimates. We can examine whether a population is likely to adapt and make relative comparisons

444 between stress treatments, i.e. identify those where we expect the largest relative increase in fitness. We thus

445 show that existing quantitative genetic tools are informative over time scales beyond single generation

446 responses. However, reliability of predictions may require a fully integrative approach including potential

447 genetic covariances between the sexes as well as a careful choice of the traits used as fitness estimate.

$449 \quad$ Evolutionary potential and adaptation

$450 \quad V_{A}$ of female fitness was higher than $V_{A}$ of male fitness in all conditions except Hot-Dry. Egg production is likely

451 to be costly and reduced when females are stressed due to a resource allocation trade-off. It is therefore not

452 surprising that variation in reproductive output could be mainly explained by genetic differences among

453 females, whereas genetic variation among males had only a minor influence. However, this changed when

454 humidity was reduced. In Dry conditions, $\mathrm{V}_{\mathrm{A}}$ for males became significant (but was still much smaller compared 
455 to females) and in Hot-Dry, $\mathrm{V}_{\mathrm{A}}$ in both sexes were similar. It was observed that the condition of Tribolium males

456 could have a significant influence on the reproductive output of their mating partner. Starvation of males

457 reduced insemination success (Lewis et al. 2012), the number of eggs laid, and the proportion of unfertilized

458 eggs (Sbilordo et al. 2011). It seems plausible that desiccation might have similar effects. Producing ejaculate

459 may be costly and decreased under dry conditions to reduce water loss. Genetic differences in male drought

460 resistance would then translate into observed offspring variation and result in a higher male influence on

461 female reproductive output. Although male $V_{\mathrm{A}}$ was small in ancestral conditions, evolution of males seemed to

462 play an important role in adapting to Hot-Dry. In contrast to the between-sex differences in fitness $V_{\mathrm{A}}$, we did

463 not find clear differences in $\mathrm{V}_{\mathrm{A}}$ between sexes for body size. The largest difference was in Control (Figure 2),

464 with a lower female $\mathrm{V}_{\mathrm{A}}$. It is likely that female size had been under strong positive selection since it is

465 associated with fecundity (Honěk 1993), which might explain the observed reduction. Additionally, while we

466 observed an increase in $\mathrm{V}_{\mathrm{A}}$ for male fitness in the Dry treatment, $\mathrm{V}_{\mathrm{A}}$ for male size was reduced in Dry. A drought

467 effect was thus also detectable for size: When we explored the effects of temperature and humidity on size,

468 we found a significant (although small) sex-treatment-interaction indicating that male size was more sensitive

469 to drought than female size. $\mathrm{G}$ x E for male size occurred when conditions differed in humidity.

471 Positive cross-condition genetic correlations indicated an absence of an evolutionary trade-off between

472 drought and heat adaptation. Consistently, we did not find any selection line performing worse than control

473 lines in any condition after experimental evolution. It was previously shown that the treatments induce

474 substantial changes in gene expression (Koch and Guillaume 2020). It might then be that selection in the stress

475 treatments is on genes that are not expressed in control and thus allele frequency changes do not sufficiently

476 affect fitness under control conditions. Because positive genetic correlations indicate a similar genetic basis for

477 fitness under different conditions, adapting to one condition should result in a correlated response and

478 increased fitness in other conditions. Accordingly, we found that drought adaptation improved heat resistance

479 (Figure 7). However, this correlated response was asymmetric since we did not observe an effect of heat

480 adaptation on drought resistance. A possible explanation is that selection in dry and hot conditions shifted 
481 allele frequencies of genes with different pleiotropic effects (Bohren et al. 1966). Alternatively, the pleiotropic

482 degree of a gene might be environment dependent (Barrett et al. 2009). Interestingly, we found that genetic correlations in generation one were lower when the conditions differed in both temperature and humidity, which is the case in Control-Hot-Dry and Dry-Hot (Figure 4) suggesting that the genetic basis for adaptation to heat and drought might be slightly different. treatments and a negative correlation in Hot. Several studies in the wild (Brommer et al. 2007; Foerster et al. reported negative genetic correlations between female and male fitness. It is not well understood how crosssex correlations are influenced by the environment and contrasting predictions have been made. For instance, it was argued that in a population far from its optimum after an environmental shift, females and males might experience similar directional selection leading to high a positive correlation in their fitness (Berger et al. 2014;

Taking the male $V_{A}$ and the covariances between female and male fitness into account led us to predict the highest relative increase in fitness in Hot-Dry and smaller but similar increases in Dry and Hot. Ignoring male and cross-sex effects when studying the adaptive capacity of a population can thus lead to misleading predictions. The results of the fitness assay after 20 generations mainly matched our predictions but increases

503 in fitness were much lower than our estimated upper limit. However, our predictions are based on the 504 assumption of constant $\mathrm{V}_{\mathrm{A}}$ and consequently exponentially increasing fitness (Falconer and MacKay 1996). 
506 egg laying rate, exist and should prevent an infinite fitness increase. It is also important to note that the

507 conditions during the fitness assay were not exactly the same as during evolution. To measure the offspring

508 number per female. each female was kept individually in egg-laying tubes. In contrast, all individuals of the

509 same line were kept in one vial during experimental evolution. Effects of density and competition that might

510 have influenced adaptation were not captured in our fitness assay. Our results after 19 generations might be

511 further influenced by evolution of control lines that were used as reference representing the ancestral non-

512 evolved stage. Significant $\mathrm{V}_{\mathrm{A}}$ under control conditions in addition to high and positive genetic correlations

513 between fitness in control and stress conditions suggest a fitness increase in control lines over time and

514 correlated responses in the treatments. This might have led to an underestimation of the true fitness increase

515 of adapted selection lines. Interestingly, we found no difference between lines from different selection

516 regimes in Control. A reason might be that genes responsible for fitness in the treatments are not expressed

517 under control conditions. Evolutionary changes in these genes that occurred during adaptation to the

518 treatments would not show any effect on fitness under control conditions leading to cryptic genetic variation.

519 In control lines these genes were not exposed to selection and consequently control lines showed a lower

520 fitness compared to adapted selection line when exposed to the treatments.

\section{1}

\section{$522 \quad$ Variance components of fitness and body size}

523 Both traits differed markedly in the proportion of different variance components and in the environmental 524 effect on genetic variances. $\mathrm{h}^{2}$ was much higher in size than in fitness. This is a common finding in many studies

525 (Roff and Mousseau 1987; Kruuk et al. 2000; McCleery et al. 2004; Teplitsky et al. 2009). It had been initially 526 interpreted as evidence for strong selection depleting $\mathrm{V}_{\mathrm{A}}$ in fitness related traits. However, a high $\mathrm{V}_{\mathrm{A}}$ can be 527 concealed in $\mathrm{h}^{2}$ by a simultaneous increase of the total variance (Houle 1992; Merilä and Sheldon 1999;

$528 \quad$ Hansen et al. 2011; Wheelwright et al. 2014). Given the highly polygenic nature of fitness, it was even argued

529 that fitness might show higher $\mathrm{V}_{\mathrm{A}}$ (Merilä and Sheldon 1999), since it represents a larger mutational target.

530 Furthermore, fitness is a composite character with a high number of morphological, physiological and 531 behavioral traits contributing to it, each of them with some underlying genetic variance. However, each 
532 contributing trait may increase the influence of environmental variation, leading to a higher total variance in

533 fitness (Price and Schulter 1991) and thus a lower $h^{2}$. According to those previous considerations, we found

534 much higher estimates of mean-scaled $V_{A}\left(C_{A}, I_{A}\right)$ in offspring number than in body size and a lower $h^{2}$ in

535 fitness. The lower $\mathrm{h}^{2}$ was mainly due to a higher proportion of environmental variance $\left(\mathrm{V}_{\mathrm{R}}\right)$ when compared to

536 body size. Contrary to body size, we could not directly estimate $V_{D}$ for fitness because we could not

537 disentangle $V_{D}$ from $V_{M}$ and common environmental effects in the $F 1$ with our half-sib/full-sib breeding design.

538 However, if $V_{D}$ were present, then it should be included in $V_{M}$, because it contributes to full-sib resemblance

539 via a shared mother. Comparative studies investigating the relative amount of $V_{A}$ and $V_{D}$ suggested that

540 proportion of $V_{D}$ can be substantial and of the same magnitude as $\mathrm{V}_{\mathrm{A}}$ (Crnokrak and Roff 1995; Wolak and

541 Keller 2014). An increased proportion of $V_{D}$ is expected in populations under strong selection, since $V_{D}$ is not

542 affected by natural selection (Crnokrak and Roff 1995; Merilä and Sheldon 1999; Roff and Emerson 2006;

543 Sztepanacz and Blows 2015), or with increased inbreeding (Falconer and Makay, 1996). In our experiment, $V_{M}$

544 for fitness was much lower than $\mathrm{V}_{\mathrm{A}}$, even close to zero in Hot and Hot-Dry (Figure 3), suggesting that $\mathrm{V}_{\mathrm{D}}$

545 contributed much less to total genetic variance than $\mathrm{V}_{\mathrm{A}}$.

\section{6}

547 We could directly estimate $V_{D}$ for body size in the F2 cross and can thus provide data for this rarely estimated

548 variance component. Although our $\mathrm{V}_{\mathrm{D}}$ estimates for size were always associated with large SE, they suggest

549 that $V_{D}$ is present and environment dependent. The highest estimates of $V_{D}$ were found in Dry and Hot for both

550 male and female size, while it remained close to zero in Control and Hot-Dry. This environmental dependence

551 of $V_{D}$ has not been studied in detail before, although the environmental dependence of inbreeding depression

552 was previously described (Bijlsma et al. 1999; Armbruster and Reed 2005; Fox and Reed 2011). Both $V_{D}$ and

553 inbreeding depression are expected to increase with inbreeding, for instance in shrinking populations when

554 allele and genotype frequencies change because of increased drift (Falconer and Makay, 1996). This could have

555 important implications since environmental changes may emphasize the effects of inbreeding on survival and

556 thus on population size too, directly affecting genetic variances. 
558 The large uncertainty around our estimates of $\mathrm{V}_{\mathrm{D}}$ may come from the double-first cousin (DFC) breeding design

559 used in the F2, despite the large sample size we had (827 family pairs with DFC offspring). Although the DFC

560 design was proposed to estimate $V_{D}$ (Fairbairn and Roff 2006), it may have limited statistical power because

561 the probability that DFC share alleles identical by descent at a given locus is only $6.25 \%$, resulting in large SE,

562 in contrast to full-sibs where this probability is $25 \%$. Therefore, comparing maternal half-sibs and full-sibs

563 might be a much more powerful approach to estimate $V_{D}$, while it allows us to disentangle non-additive from

564 maternal effects at the same time.

565

566 Although we could estimate genetic correlations between fitness and size with our data set (Supplemental

567 Table S4), it was not possible to get unbiased estimates of those correlations. With our design, we could not

568 rule out a strong confounding effect of population density because body size was measured in the F2, the

569 offspring of beetles used for the fitness assay (Supplement Figure S1). Consequently, offspring of females with

570 a high fitness (i.e. high offspring number) grew at a higher density since we used identical tubes with the same

571 amount of flour for all females in the fitness assay.

\section{$573 \quad$ Conclusions}

574 Our study showed an increased adaptive potential in stressful conditions and a corresponding adaptation to 575 those conditions after 20 generations. Although precise predictions of relative increase in fitness were not 576 possible over this time period, we could make correct qualitative predictions. We expected and observed the 577 highest relative fitness increase in the most stressful hot-dry condition and similar increases in single stress 578 treatments. The apparently high adaptive potential of female beetles in Hot was limited by a negative genetic 579 correlation with male fitness. We further found that genetic effects of males on fitness can be large and can 580 increase the adaptive potential. Comparing genetic variances of fitness and size showed that they differed in 581 their variance composition and in their cross-sex and cross-environment genetic covariances. Environmental 582 effects on genetic variances were also not consistent between the two traits. We thus advise caution if studies 
583 interested in fitness $\mathrm{V}_{\mathrm{A}}$ and the adaptive capacity of a population use body size as a proxy. Overall, we found

584 that genetic variance in fitness is a key estimate of a population's adaptive capacity for time scales over 20

585 generations. As such, it may help predict the adaptive response of populations exposed to new environmental

586 conditions and help identify the populations most at risk of extinction. However, the reliability of such

587 predictions will depend on the fitness estimate chosen and on the full integration of the multifaceted aspects

588 of adaptation. Inclusion of genetic covariances between female and male fitness and genotype by environment

589 interactions is thus important.

591 Author contributions: FG and ELK designed the experiment. ELK and SHS conducted the experiment (crossing

592 and fitness assay). SHS performed size measurements. ELK analysed the data. FG and ELK wrote the

593 manuscript. SHS contributed and commented to manuscript.

595 Acknowledgements: This work was supported by the Swiss National Science Foundation, grants

596 PPOOP3_1144846 and PP00P3_176965 to FG. We would like to thank all people helping with fitness

597 measurements: Julian Bauer, Valérian Zeender, Maria Domenica Moccia, Sara Meier, Cilgia Lippuner, Tim

598 Schoch, Tim Emmenegger, Elke Karaus.

\section{$600 \quad$ References}

601 Armbruster, P., and D. H. Reed. 2005. Inbreeding depression in benign and stressful environments. Heredity

$60295: 235-242$.

603 Arnold, P. A., A. B. Nicotra, and L. E. B. Kruuk. 2019. Sparse evidence for selection on phenotypic plasticity in 604 response to temperature. Philos. Trans. R. Soc. B Biol. Sci. 374.

605 Barrett, R. D. H., S. M. Rogers, and D. Schluter. 2009. Environment specific pleiotropy facilitates divergence at 606 the ectodysplasin locus in threespine stickleback. Evolution 63:2831-2837.

607 Bates, D., M. Mächler, B. Bolker, and S. Walker. 2015. Fitting linear mixed-effects models using Ime4. J. Stat. 608 Softw. 67. 
Bell, G., and A. Gonzalez. 2009. Evolutionary rescue can prevent extinction following environmental change.

Ecol. Lett. 12:942-948.

Berger, D., K. Grieshop, M. I. Lind, J. Goenaga, A. A. Maklakov, and G. Arnqvist. 2014. Intralocus sexual conflict and environmental stress. Evolution 68:2184-2196. and purging in Drosophila melanogaster. J. Evol. Biol. 12:1125-1137.

Bijma, P., and M. J. Wade. 2008. The joint effects of kin, multilevel selection and indirect genetic effects on response to genetic selection. J. Evol. Biol. 21:1175-1188.

Bohren, B. B., W. G. Hill, and A. Robertson. 1966. Some observations on asymmetrical correlated responses to selection. Genet. Res. 7:44-57.

619 Bonduriansky, R., and S. F. Chenoweth. 2009. Intralocus sexual conflict. Trends Ecol. Evol. 24:280-288.

620 Brommer, J. E., M. Kirkpatrick, A. Qvamström, and L. Gustafsson. 2007. The intersexual genetic correlation for $621 \quad$ lifetime fitness in the wild and its implications for sexual selection. PLoS One 2:e744. Public Library of Science.

Bubliy, O. A., T. N. Kristensen, V. Kellermann, and V. Loeschcke. 2012. Plastic responses to four environmental stresses and cross-resistance in a laboratory population of Drosophila melanogaster. Funct. Ecol. 26:245-

\section{6} 253.

Bubliy, O. A., and V. Loeschcke. 2005. Correlated responses to selection for stress resistance and longevity in a laboratory population of Drosophila melanogaster. J. Evol. Biol. 18:789-803.

Butler, D. G., B. R. Cullis, A. R. Gilmour, and B. J. Gogel. 2009. Analysis of mixed models for S language

633 Charmantier, A., and D. Garant. 2005. Environmental quality and evolutionary potential: lessons from wild populations. Proc. R. Soc. B Biol. Sci. 272:1415-1425.

635 Charmantier, A., D. Garant, and L. E. B. Kruuk (eds). 2014. Quantitative genetics in the wild. Oxford University 
Press, Oxford.

Charmantier, A., R. H. McCleery, L. R. Cole, C. Perrins, L. E. B. Kruuk, and B. C. Sheldon. 2008. Adaptive

phenotypic plasticity in response to climate change in a wild bird population. Science 320:800-803.

Collet, J. M., S. Fuentes, J. Hesketh, M. S. Hill, P. Innocenti, E. H. Morrow, K. Fowler, and M. Reuter. 2016. Rapid 795.

Connallon, T., and M. D. Hall. 2016. Genetic correlations and sex-specific adaptation in changing environments. Evolution 70:2186-2198.

\section{4}

Crnokrak, P., and D. A. Roff. 1995. Dominance variance: Associations with selection and fitness. Heredity

de Villemereuil, P. 2018. Quantitative genetic methods depending on the nature of the phenotypic trait. Ann.

N. Y. Acad. Sci. 1422:29-47.

Delcourt, M., M. W. Blows, and H. D. Rundle. 2009. Sexually antagonistic genetic variance for fitness in an ancestral and a novel environment. Proc. R. Soc. B Biol. Sci. 276:2009-2014.

DeWitt, T. J., A. Sih, and D. S. Wilson. 1998. Costs and limits of phenotypic plasticity. Trends Ecol. Evol. 13:77-

81.

Duputié, A., A. Rutschmann, O. Ronce, and I. Chuine. 2015. Phenological plasticity will not help all species adapt to climate change. Glob. Chang. Biol. 21:3062-3073.

Etterson, J. R., and R. G. Shaw. 2001. Constraint to adaptive evolution in response to global warming. Science

Fairbairn, D. J., and D. A. Roff. 2006. The quantitative genetics of sexual dimorphism: Assessing the importance of sex-linkage. Heredity 97:319-328. Essex.

Fisher, R. A. 1930. The genetical theory of natural selection. Clarendon Press, Oxford, UK. 

antagonistic genetic variation for fitness in red deer. Nature 447:1107-1110. study and meta-analysis. Evolution 65:246-258. Sci. U. S. A. 115:10070-10075.

Garcia-Gonzalez, F., L. W. Simmons, J. L. Tomkins, J. S. Kotiaho, and J. P. Evans. 2012. Comparing evolvabilities: International Ltd, Hemel Hempstead, UK. Rev. Ecol. Evol. Syst. 49:457-476.

Hereford, J. 2009. A Quantitative Survey of Local Adaptation and Fitness Trade-Offs. Am. Nat. 173:579-588. conditions. Trends Ecol. Evol. 14:96-101.

Honěk, A. 1993. Intraspecific Variation in Body Size and Fecundity in Insects : A General Relationship. Nord. Soc. Oikos 66:483-492.

Houle, D. 1992. Comparing Evolvability and Variability. Genetics 130:195-204.

Husby, A., M. E. Visser, and L. E. B. Kruuk. 2011. Speeding up microevolution: The effects of increasing temperature on selection and genetic variance in a wild bird population. PLoS Biol. 9:e1000585. 
Kellermann, V. M., B. van Heerwaarden, A. A. Hoffmann, and C. M. Sgrò. 2006. Very Low Additive Genetic Variance and Evolutionary Potential in Multiple. Evolution 60:1104-1108. multiple stress in Tribolium castaneum. PLoS Genet. 16:e1008768.

Kruuk, L. E. B. 2004. Estimating genetic parameters in natural populations using the "animal model." Philos. Trans. R. Soc. B Biol. Sci. 359:873-890. The Royal Society. Heritability of fitness in a wild mammal population. Proc. Natl. Acad. Sci. 97:698-703.

Lande, R. 1979. Quantitative genetic analysis of multivariate evolution, applied to brain: body size allometry. Evolution 33:402-416. Wiley/Blackwell (10.1111). reproductive performance in Tribolium flour beetles. J. Evol. Biol. 25:438-451. fecundity in a locally adapted Drosophila melanogaster population. J. Evol. Biol. 22:637-643.

McCleery, R. H., R. A. Pettifor, P. Armbruster, K. Meyer, B. C. Sheldon, and C. M. Perrins. 2004. Components of Variance Underlying Fitness in a Natural Population of the Great Tit Parus major. Am. Nat. 164:E62-E72.

McFarlane, S. E., J. C. Gorrell, D. W. Coltman, M. M. Humphries, S. Boutin, and A. G. Mcadam. 2014. Very low 

Bacterial Oral Infections. PLoS One 8. frequency-dependent selection of sexually antagonistic alleles in Myodes glareolus. Science 334:972974. American Association for the Advancement of Science.

Morrissey, M. B., and T. Bonnet. 2019. Analogues of the fundamental and secondary theorems of selection, assuming a log-normal distribution of expected fitness. J. Hered. 110:396-402. Oxford Academic.

Mousseau, T. A., and D. A. Roff. 1987. Natural selection and the heritability of fitness components. Heredity 59:181-197.

Parmesan, C. 2006. Ecological and Evolutionary Responses to Recent Climate Change. Annu. Rev. Ecol. Evol. Syst. 37:637-669.

Pfennig, D. W., M. A. Wund, E. C. Snell-Rood, T. Cruickshank, C. D. Schlichting, and A. P. Moczek. 2010. Phenotypic plasticity's impacts on diversification and speciation. Trends Ecol. Evol. 25:459-467.

Poissant, J., A. J. Wilson, and D. W. Coltman. 2010. Sex-specific genetic variance and the evolution of sexual dimorphism: A systematic review of cross-sex genetic correlations. Evolution 64:97-107.

Price, G. R. 1972. Fisher's 'fundamental theorem' made clear. Ann. Hum. Genet. 36:129-140.

Price, T., and D. Schulter. 1991. On the Low Heritability of Life-History Traits. Evolution 45:853-861.

Punzalan, D., M. Delcourt, and H. D. Rundle. 2014. Comparing the intersex genetic correlation for fitness across

743 Roff, D. A., and K. Emerson. 2006. Epistasis and dominance: evidence for differential effects in life-history 
versus morphological traits. Evolution 60:1981.

745 Roff, D. A., and T. A. Mousseau. 1987. Quantitative genetics and fitness: lessons from Drosophila. Heredity

$746 \quad 58: 103-118$.

747 Rowiński, P. K., and B. Rogell. 2017. Environmental stress correlates with increases in both genetic and residual $748 \quad$ variances: A meta-analysis of animal studies. Evolution 71:1339-1351.

749 Sbilordo, S. H., V. M. Grazer, M. Demont, and O. Y. Martin. 2011. Impacts of starvation on male reproductive 750 success in Tribolium castaneum. Evol. Ecol. Res. 13:347-359.

751 Schluter, D., T. D. Price, and L. Rowe. 1991. Conflicting selection pressures and life history trade-offs.

752 Sgrò, C. M., and A. A. Hoffmann. 1998. Effects of stress combinations on the expression of additive genetic 753 variation for fecundity in Drosophila melanogaster. Genet. Res. 72:13-18.

754 Sgrò, C. M., and A. A. Hoffmann. 2004. Genetic correlations, tradeoffs and environmental variation. Heredity $75593: 241-248$.

756 Shaw, R. G., and J. R. Etterson. 2012. Rapid climate change and the rate of adaptation: Insight from $757 \quad$ experimental quantitative genetics.

758 Shaw, R. G., and F. H. Shaw. 2014. Quantitative genetic study of the adaptive process. Heredity 112:13-20.

759 Sikkink, K. L., R. M. Reynolds, W. A. Cresko, and P. C. Phillips. 2015. Environmentally induced changes in 760 correlated responses to selection reveal variable pleiotropy across a complex genetic network. Evolution $761 \quad 69: 1128-1142$.

762 Simons, A. M., and D. A. Roff. 1996. The Effect of a Variable Environment on the Genetic Correlation Structure 763 in a Field Cricket. Evolution 50:267-275.

764 Snell-Rood, E. C., J. D. Van Dyken, T. Cruickshank, M. J. Wade, and A. P. Moczek. 2010. Toward a population 765 genetic framework of developmental evolution: The costs, limits, and consequences of phenotypic $766 \quad$ plasticity.

767 Sokolova, I. M., M. Frederich, R. Bagwe, G. Lannig, and A. A. Sukhotin. 2012. Energy homeostasis as an 768 integrative tool for assessing limits of environmental stress tolerance in aquatic invertebrates. Mar. $769 \quad$ Environ. Res. 79:1-15.

770 Sztepanacz, J. L., and M. W. Blows. 2015. Dominance genetic variance for traits under directional selection in 
Drosophila serrata. Genetics 200:371-384.

Teplitsky, C., J. A. Mills, J. W. Yarrall, and J. Merilä. 2009. Heritability of fitness components in a wild bird population. Evolution 63:716-726.

Thomas, C. D., A. Cameron, R. E. Green, M. Bakkenes, L. J. Beaumont, Y. C. Collingham, B. F. N. N. Erasmus, M. Ferreira De Siqueira, A. Grainger, L. Hannah, L. Hughes, B. Huntley, A. S. Van Jaarsveld, G. F. Midgley, L. climate change. 427:145-148.

Valladares, F., E. Gianoli, and J. M. Gómez. 2007. Ecological limits to plant phenotypic plasticity. New Phytol. $176: 749-763$.

Waldvogel, A.-M., B. Feldmeyer, G. Rolshausen, M. Exposito-Alonso, C. Rellstab, R. Kofler, T. Mock, K. Schmid,

Wheelwright, N. T., L. F. Keller, and E. Postma. 2014. The effect of trait type and strength of selection on heritability and evolvability in an island bird population. Evolution 68:3325-3336.

Wickman, P.-O., and B. Karlsson. 1989. Abdomen Size, Body Size and the Reproductive Effort of Insects. Oikos 56:209.

Wilson, A. J., J. M. Pemberton, J. G. Pilkington, D. W. Coltman, D. V Mifsud, T. H. Clutton-Brock, and L. E. B. Kruuk. 2006. Environmental coupling of selection and heritability limits evolution. PLoS Biol. 4:12701275.

Wilson, A. J., D. Réale, M. N. Clements, M. M. Morrissey, E. Postma, C. A. Walling, L. E. B. Kruuk, and D. H. Nussey. 2010. An ecologist's guide to the animal model. J. Anim. Ecol. 79:13-26.

Wolak, M. E. 2012. Nadiv: An R package to create relatedness matrices for estimating non-additive genetic variances in animal models. Methods Ecol. Evol. 3:792-796.

Wolak, M. E., P. Arcese, L. F. Keller, P. Nietlisbach, and J. M. Reid. 2018. Sex-specific additive genetic variances and correlations for fitness in a song sparrow (Melospiza melodia) population subject to natural immigration and inbreeding. Evolution 72:2057-2075. 
bioRxiv preprint doi: https://doi.org/10.1101/2020.02.26.966119; this version posted July 15, 2020. The copyright holder for this preprint (which

was not certified by peer review) is the author/funder, who has granted bioRxiv a license to display the preprint in perpetuity. It is made available under aCC-BY-NC-ND 4.0 International license.

798 Wolak, M. E., and L. F. Keller. 2014. Dominance genetic variance and inbreeding in natural populations. Pp.

799 104-127 in A. Charmantier and L. E. B. Kruuk, eds. Quantitative Genetics in the Wild. Ox, Oxford, UK.

800 Wood, C. W., and E. D. Brodie. 2015. Environmental effects on the structure of the G-matrix. Evolution

$801 \quad 69: 2927-2940$.

802 Wood, C. W., and E. D. Brodie. 2016. Evolutionary response when selection and genetic variation covary across

803 environments. Ecol. Lett. 19:1189-1200. 